\title{
Local honokiol application inhibits intimal thickening in rabbits following carotid artery balloon injury
}

\author{
YU WANG $^{1 *}$, DANYANG ZHAO ${ }^{2 *}, \mathrm{JING} \mathrm{SHENG}^{1}$ and PING LU ${ }^{1}$ \\ Departments of ${ }^{1}$ Geriatrics and ${ }^{2}$ Plastic and Reconstructive Surgery, Shanghai Ninth People's Hospital, \\ Shanghai Jiao Tong University School of Medicine, Shanghai 200011, P.R. China
}

Received November 13, 2016; Accepted July 24, 2017

DOI: $10.3892 / \mathrm{mmr} .2017 .8076$

\begin{abstract}
Honokiol is a natural bioactive product with anti-tumor, anti-inflammatory, anti-oxidative, anti-angiogenic and neuroprotective properties. The present study aimed to investigate the effects of honokiol treatment on intimal thickening following vascular balloon injury. The current study determined that perivascular honokiol application reduced intimal thickening in rabbits 14 days after carotid artery injury, it may inhibit vascular smooth muscle cell (VSMCs) proliferation and reduce collagen deposition in local arteries. The findings of the presents study also suggested that honikiol may increase the mRNA expression levels of matrix metalloproteinase-1 (MMP-1), MMP-2 and MMP-9 and decrease tissue inhibitor of metalloproteinase-1 (TIMP-1) mRNA expression in the rabbit arteries. Additionally, perivascular honokiol application inhibited intimal thickening, possibly via inhibition of the phosphorylation of SMAD family member $2 / 3$.
\end{abstract}

\section{Introduction}

Intimal hyperplasia contributes to the pathological process of several vascular disorders, including restenosis following angioplasty, transplant vasculopathy, vein graft stenosis and atherosclerosis (1). During vascular remodeling, vascular smooth muscle cells (VSMCs) proliferate, migrate and change from a contractile phenotype to a synthetic phenotype (2), inhibition of VSMC proliferation, migration and phenotypic modulation is a feasible treatment strategy to prevent intimal

Correspondence to: Dr Jing Sheng or Dr Ping Lu, Department of Geriatrics, Shanghai Ninth People's Hospital, Shanghai Jiao Tong University School of Medicine, 639 Zhizaoju Road, Shanghai 200011, P.R. China

E-mail: 15000023010@163.com

E-mail: wobushifeizao@163.com

${ }^{*}$ Contributed equally

Key words: honokiol, intimal thickening, vascular smooth muscle cells, matrix metalloproteinase, phosphorylated-Smad2/3 hyperplasia. The extracellular matrix (ECM) is composed of collagen subtypes and proteoglycans and has an important role in the process of intimal thickening. Collagen is the most abundant matrix protein in ECM, regulating collagen synthesis and degradation may also be a therapeutic target for intimal hyperplasia (3).

Honokiol (3',5-di-(2-propenyl)-1,1'-biphenyl-2,4'-diol) (Fig. 1A) is a natural bioactive product obtained from plants in the genus Magnolia and has been determined to have multiple biological activities, including anti-cancer, anti-angiogenic, anti-inflammatory, anti-hypertensive, neuroprotective, anti-oxidative and anti-thrombosis properties (4-7). Honokiol has been shown to have the ability to protect endothelial cells and stimulate prostacyclin release in a rat carotid thrombosis model (6), which was revealed to have potential in preventing intimal hyperplasia $(8,9)$. Lee et al determined that honokiol treatment inhibited VSMC growth by activating p38 mitogen activated protein kinase (10). A previous study suggested that honokiol may suppress tumor necrosis factor-a $(\mathrm{TNF}-\alpha)$-induced rat aortic smooth muscle cell proliferation via caspase and mitochondria-dependent apoptosis (11). These studies revealed that honokiol may have therapeutic potential in the prevention of vascular remodeling in cardiovascular diseases.

The present study investigated the effect of honokiol on intimal thickening following vascular balloon injury. Perivascular drug/gene delivery may effectively prevent intimal hyperplasia in preclinical experiments $(12,13)$. Therefore, the present study hypothesized that perivascular honokiol application may inhibit neointimal hyperplasia in rabbits following carotid artery balloon injury through anti-proliferative ability. The present study investigated the effect of perivascular honokiol application on the expression of collagen, matrix metalloproteinases (MMPs), the tissue inhibitor of metalloproteinase-1 (TIMP-1) and the underlying mechanism involved in this process.

\section{Materials and methods}

Materials. F-127 pluronic gel was purchased from Sigma-Aldrich; Merck Millipore (Darmstadt, Germany). Honokiol was purchased from Selleck Chemicals (Houston, TX, USA). 3F Fogarty embolectomy catheters were obtained from Edwards Lifesciences (Irvine, CA, USA). The primer 
sequences used in mRNA analysis are presented in Table I. The primary antibodies used in western blotting analysis were: Smad2/3 (Santa Cruz Biotechnology, Inc., Dallas, TX, USA; catalog no. sc-6032), phosphorylated-Smad2/3 (p-Smad2/3; Santa Cruz Biotechnology, Inc.; catalog no. sc-11769), transforming growth factor- $\beta 1$ (TGF- $\beta 1$; Novus Biologicals, LLC, Littleton, CO, USA; catalog no. MAB240), TGF- $\beta$ receptor type I (TGF- $\beta$ RI; Santa Cruz Biotechnology, Inc.; catalog no. sc-398-G), TGF- $\beta$ RII (Santa Cruz Biotechnology, Inc.; catalog no. sc-17792) and GAPDH (Abcam, Cambridge, UK; catalog no. ab8245). Mouse monoclonal $\alpha$-smooth muscle actin ( $\alpha$-SMA; Abcam; catalog no. ab7817) and rabbit monoclonal anti-proliferating cell nuclear antigen (PCNA; Abcam; catalog no. ab19166) were used for immunohistochemistry analysis. The secondary antibodies were: Mouse anti-goat IgG horse radish peroxidase (HRP) (Santa Cruz Biotechnology, Inc.; catalog no. sc-2354) (for Smad2/3, p-Smad2/3, TGF- $\beta R I$ ), goat anti-mouse IgG HRP (Abcam; catalog no. ab205719) (for TGF- $\beta 1$, TGF- $\beta$ RII, GAPDH and a-SMA), goat anti-rabbit IgG HRP (Abcam; catalog no. ab205718) (for PCNA). Remaining solvents and reagents were analytical grade.

Preparation of the honokiol-containing pluronic gel. F-127 pluronic gel $(30 \% \mathrm{w} / \mathrm{v})$ in saline was prepared and stored at $-20^{\circ} \mathrm{C}$ for future use. Honokiol $(10 \mathrm{mg})$ was dissolved in $1 \mathrm{ml}$ dimethylsulphoxide (DMSO) and honokiol-containing pluronic gel was prepared by adding $1 \mathrm{ml}$ of this solution to $9 \mathrm{ml}$ of the previously prepared $30 \%$ pluronic gel at $4^{\circ} \mathrm{C}$. Therefore, $1 \mathrm{ml}$ of this solution contained $1 \mathrm{mg}$ of honokiol. The resulting honokiol-containing pluronic gel was stored at $-20^{\circ} \mathrm{C}$ for future use. It is of note that DMSO was used for the dilution because honokiol is insoluble in water and has a relatively poor solubility in ethanol.

Balloon endothelial denudation in rabbit carotid artery and perivascular honokiol application. Male New Zealand White rabbits (3.0-3.5 kg, $\mathrm{n}=24$, aged 4-5 months) were obtained from the Laboratory Animal Center of The Ninth People's Hospital, Shanghai Jiao Tong University School of Medicine (Shanghai, China), animals were housed at a temperature of $23 \pm 1^{\circ} \mathrm{C}$ and a humidity of $50 \pm 20 \%$ for 1 week with free access to food and water. General anesthesia was induced by intravenous injection of $3 \%$ pentobarbital sodium $(1 \mathrm{ml} / \mathrm{kg})$. A midline neck incision was made and the left carotid artery (left common carotid artery, left external carotid artery, left internal carotid artery) was exposed. Subsequently, a permanent ligature was made on the left external carotid artery $\sim 5 \mathrm{~mm}$ away from the bifurcation, the left internal carotid artery was ligated by a bulldog clamp, another bulldog clamp was also placed on the proximal end of left common carotid artery. A 3F Fogarty embolectomy catheter was then introduced into the left common carotid artery through left external carotid artery to establish the animal model of vascular balloon injury. The balloon catheter was inflated with $\sim 0.1 \mathrm{ml}$ saline solution three times to denude the aorta endothelium, the length was about $3 \mathrm{~cm}$. Immediately following balloon injury, $1 \mathrm{ml}$ Pluronic gel containing honokiol or DMSO was applied around the injured carotid artery. Rabbits were randomly divided into three experimental groups: i) Honokiol treated group (1 mg, $\mathrm{n}=8$ ); ii) vehicle (equal quantity of Pluronic gel containing DMSO) treated group (vehicle alone, $n=8$ ); and iii) untreated group (balloon injury without any treatment, $n=8$ ). The left external carotid artery was ligated immediately after treatment, the bulldog clamps were removed and the incision was closed. All animals recovered after 40-60 min surgery and showed no symptoms of stroke. Animals were euthanized and sacrificed in order to collect the carotid arteries 14 days post-surgery, three segments were cut from each artery, one segment was fixed in $10 \%$ buffered formalin and the remaining two segments were stored in liquid nitrogen. The protocols were approved by the Animal Care and Use Committee of Shanghai Jiao Tong University School of Medicine.

Histopathological staining and morphometry. Carotid arteries were fixed in formalin fixative solution for $24 \mathrm{~h}$, then dehydrated in graded ethanol and subsequently embedded in paraffin. Transverse $5 \mu \mathrm{m}$ slices were cut and then stained with hematoxylin and eosin (H\&E) (3 min for hematoxylin and $30 \mathrm{sec}$ for eosin at room temperature) and Masson (5-10 min at room temperature) following the manufacturer's protocol. The cross-sectional intima area, media area and intima-to-media (I/M) area ratio were calculated from the H\&E stain slices, collagen fibers were calculated on Masson staining slices (photographed by a Nikon light microscope ). All the data was analyzed and calculated using the Image-Pro Plus version 6.0 (Media Cybernetics, Inc., Rockville, MD, USA).

Immunohistochemistry staining. Paraffin-embedded tissues were cut into 5- $\mu \mathrm{m}$ thick slices, the sections were incubated with primary antibodies ( $\alpha$-SMA, 1:2,000 dilution; and PCNA, $1: 2,000$ dilution) at $4^{\circ} \mathrm{C}$ overnight and then stained with a labeled DAB Detection kit (Fuzhou Maixin Biotech Co., Ltd., Fuzhou, China), followed by counterstaining with hematoxylin (30 sec at room temperature). Negative control was treated with PBS. A total of 5 sections of each carotid artery were selected for quantification, and 4 random fields were imaged at magnification of $\mathrm{x} 200$ in each section. The number of positively stained cells was quantified using Image-Pro PLUS version 6.0 (Media Cybernetics, Inc.).

Reverse transcription-quantitative polymerase chain reaction analysis (RT-qPCR). TRIzol reagent (Molecular Research Center, Cincinnati, OH, USA) was used to isolate total RNA from the tissues according to the manufacturer's protocol. cDNA was synthesized from $1 \mu \mathrm{g}$ of total RNA using iScript cDNA Synthesis kit (Bio-Rad Laboratories, Inc., Hercules, CA, USA) at $42^{\circ} \mathrm{C}$ for $60 \mathrm{~min}, 70^{\circ} \mathrm{C}$ for $10 \mathrm{~min}$ and $4^{\circ} \mathrm{C}$ for storage). qPCR was performed using SYBR Premix Ex Taq kit (Takara, Bio, Inc., Otsu, Japan) on a Stratagene Mx3005 RT-qPCR thermocycler $\left(30 \mathrm{sec}\right.$ at $95^{\circ} \mathrm{C}$, followed by 50 cycles of denaturation at $95^{\circ} \mathrm{C}$ for $5 \mathrm{sec}$, annealing step at $60^{\circ} \mathrm{C}$ for $35 \mathrm{sec}$ and extension at $72^{\circ} \mathrm{C}$ for $15 \mathrm{sec}$ ). The mRNA expression levels of samples were measured using the $2^{-\Delta \Delta \mathrm{Cq}}$ method (14). The primer sequences used in the current study are presented in Table I.

Western blotting analysis. Carotid arteries were homogenized in radioimmunoprecipitation assay (RIPA) lysis buffer, the protein concentration was determined using Bicinchoninic 
Table I. Primer pairs used for quantitative polymerase chain reaction analysis in this study.

\begin{tabular}{lll}
\hline Gene & \multicolumn{1}{c}{ Forward (5'-3') } & \multicolumn{1}{c}{ Reverse $\left(5^{\prime}-3^{\prime}\right)$} \\
\hline MMP-1 & AAAGGCCAGTATGCACAGCTTTC & TTCAACCACTGGGCCACTATTTC \\
MMP-2 & GAAGAGCGTGAAGGTTGGAA & TATCAGGTGGGGGTGAGAAG \\
MMP-9 & TGAGCTTTGACATCCTGCAC & TTTGTATCCGGCAAACTGGT \\
TIMP-1 & TTCTCATCGCTGGACAACTG & AGCGTAGGTCTTGGTGAAGC \\
GAPDH & GCACCGTCAAGGCTGAGAAC & TGGTGAAGACGCCAGTGGA \\
\hline
\end{tabular}

MMP, matrix metalloproteinase; TIMP-1, tissue inhibitor of metalloproteinase-1.

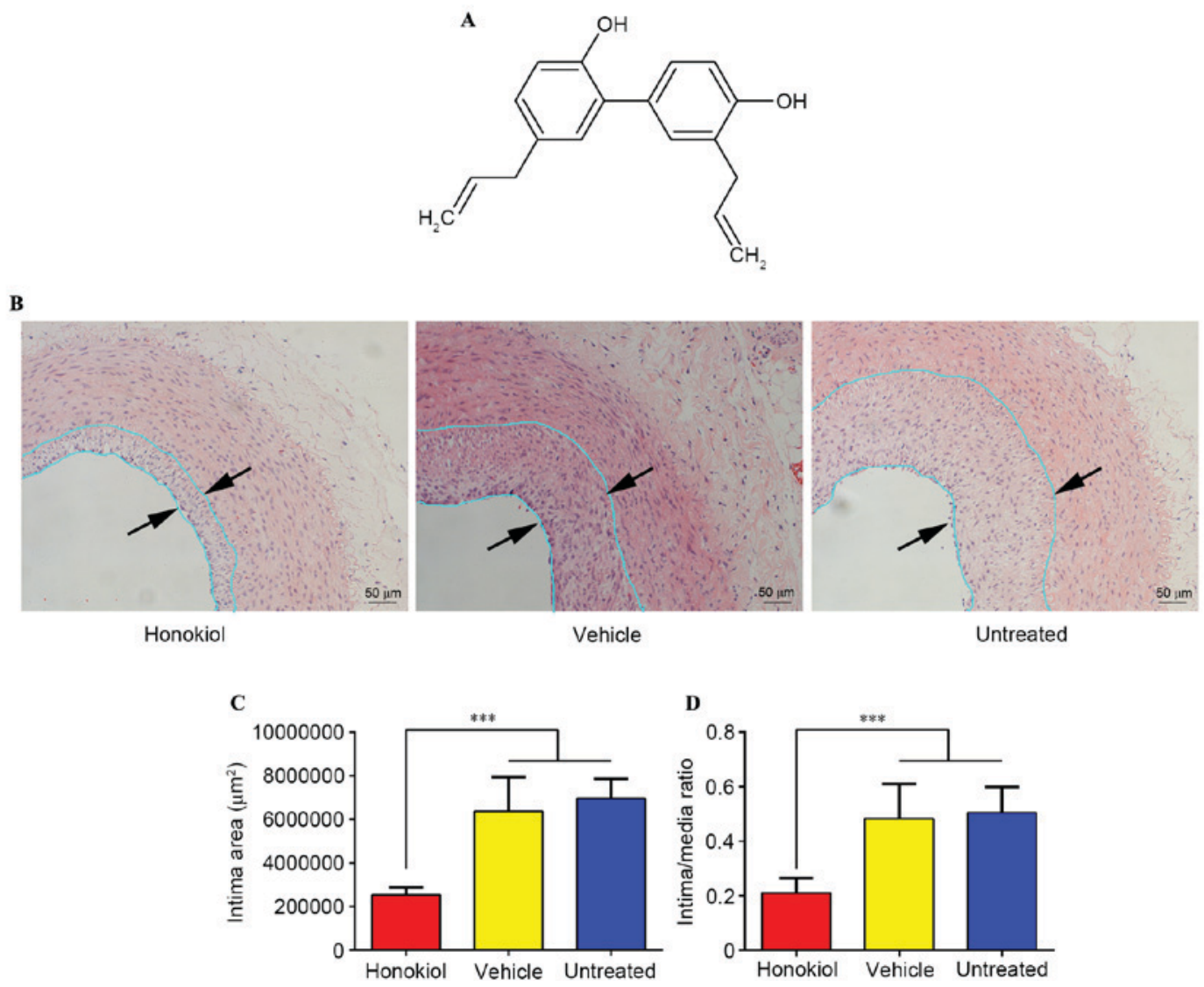

Figure 1. (A) Chemical structure of honokiol. (B) Representative hematoxylin and eosin sections of injured arteries 14 days after balloon angioplasty. The distance between arrows indicated the intima area. Magnification, $\mathrm{x} 200$. (C) Average intima area for the three treatment groups $(\mathrm{n}=8){ }^{* * * *} \mathrm{P}<0.001$. (D) Average intima/media area ratios for the three treatment groups $(n=8) .{ }^{* * * *} \mathrm{P}<0.001$.

acid Protein Assay reagent (Thermo Fisher Scientific, Inc., Waltham, MA, USA). Equal quantity protein lysates $(30 \mu \mathrm{g})$ was separated by $10 \%$ sodium dodecyl sulfate-polyacrylamide gel electrophoresis and then transferred to polyvinylidene difluoride membrane (EMD Millipore, Billerica, MA, USA). Membranes were blocked with $5 \%$ non-fat milk for $1 \mathrm{~h}$ and subsequently incubated with primary antibodies (Smad2/3 1:1,000 dilution; $\mathrm{p}-\mathrm{Smad} 2 / 3$ 1:1,000 dilution; TGF- $\beta 1$ 1:1,000 dilution; TGF- $\beta$ RI 1:200 dilution; TGF- $\beta$ RII 1:200 dilution; GAPDH 1:1,000 dilution) at $4^{\circ} \mathrm{C}$ overnight, followed by incubation with a secondary antibody for $1 \mathrm{~h}$ (at room temperature, 1:200 dilution for all). Protein bands were detected with enhanced chemiluminescence. Protein bands were determined by Quantity One version 4.62 software (Bio-Rad Laboratories,
Inc., Hercules, CA, USA), and each band was determined three times.

Statistical analysis. Data are presented as the mean \pm standard deviation and were analyzed by one-way analysis of variance with the SPSS version 22.0.0.0 (IBM Corporation, Armonk, $\mathrm{NY}$, USA). $\mathrm{P}<0.05$ was considered to indicate statistically significant difference.

\section{Results}

Perivascular honokiol application inhibited intimal hyperplasia 14 days after vascular injury. H\&E staining was used to assess the effect of honokiol on intimal thickening following 

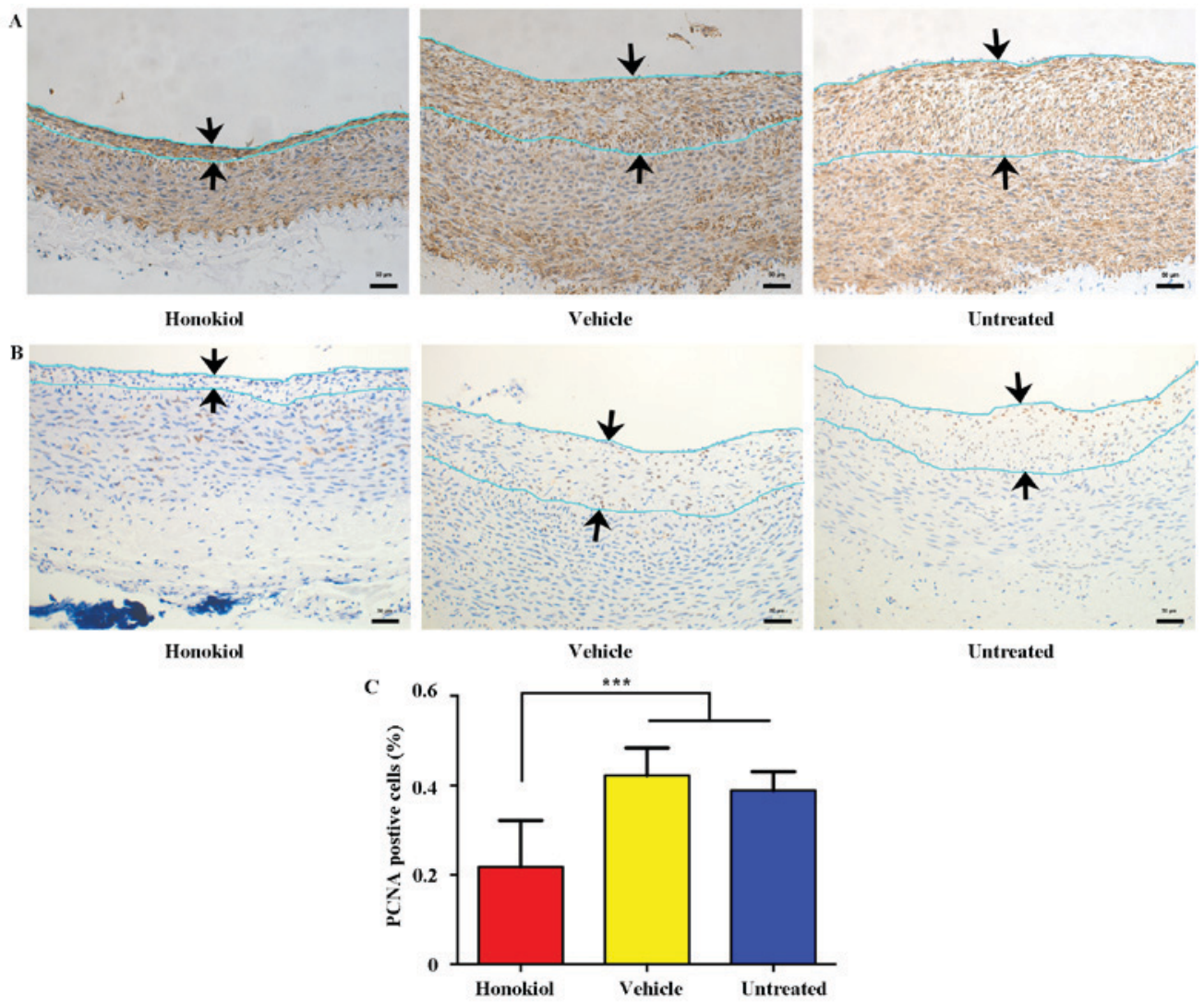

Untreated

Figure 2. Effect of perivascular honokiol application on intimal vascular smooth muscle cell proliferation. (A) Representative immunohistochemistry staining of $\alpha$-smooth muscle actin 14 days after balloon angioplasty. The distance between the arrows indicates the intima area. Magnification, x200. (B) Representative immunohistochemistry staining of PCNA 14 days after balloon angioplasty. The distance between the arrows indicates the intima area. Magnification, x200. (C) Ratio of PCNA-positive nuclei to total cell nuclei in the intima area $(\mathrm{n}=8) .{ }^{* * * *} \mathrm{P}<0.001$. PCNA, proliferating cell nuclear antigen.

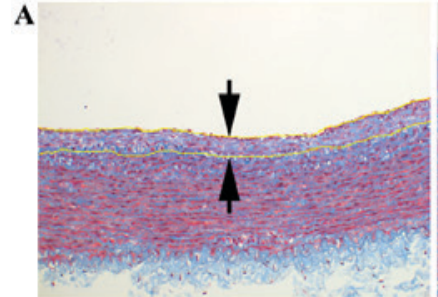

Honokiol

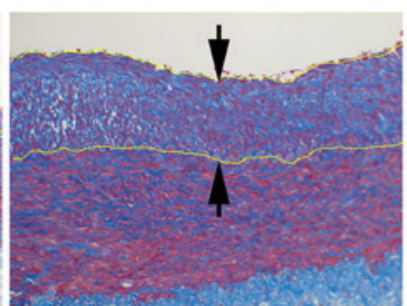

Vehicle

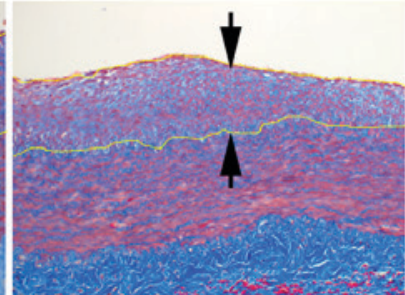

Untreated

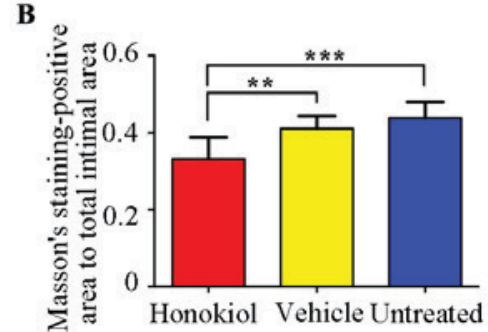

Figure 3. Effect of perivascular honokiol application on collagen deposition. (A) Representative Masson staining sections of injured arteries 14 days after balloon angioplasty. The distance between the arrows indicated the intima area. Magnifications, x200. (B) Masson's staining-positive area to total intimal area in the three injured groups $(\mathrm{n}=8) .{ }^{* *} \mathrm{P}<0.01,{ }^{* * *} \mathrm{P}<0.001$.

vascular injury, as presented in Fig. 1B. Morphometric analysis of the three treatment groups revealed that the intima area was significantly reduced in the honokiol treatment group when compared with the vehicle and untreated groups (Fig. 1C), the I/M area ratios followed a similar pattern (Fig. 1C). No significant difference was identified between intimal area and $\mathrm{I} / \mathrm{M}$ area ratios in the vehicle and the untreated groups. These findings suggested that honokiol was effective in inhibiting intimal thickening and the vehicle treatment had no effect on intimal hyperplasia.

Perivascular honokiol application reduced intimal VSMC proliferation 14 days after vascular injury. Immunohistochemistry staining for $\alpha$-SMA and PCNA was performed to investigate whether honokiol reduced neointima formation by inhibiting proliferation of VSMCs. It was determined that the majority of neointimal cells were positive for $\alpha$-SMA, indicating that the cells that proliferated in the intima were VSMCs (Fig. 2A). Additionally, intimal proliferation was observed with immunohistochemistry staining of PCNA (a marker for cell proliferation) and presented in Fig. 2B, the ratio of PCNA-positive cells was significantly reduced in the honokiol-treated group in comparison with vehicle and untreated groups (Fig. 2C). These findings indicated that local honokiol treatment significantly inhibited intimal VSMCs proliferation.

Perivascular honokiol application reduced collagen deposition following vascular injury. Collagen contents in 
A
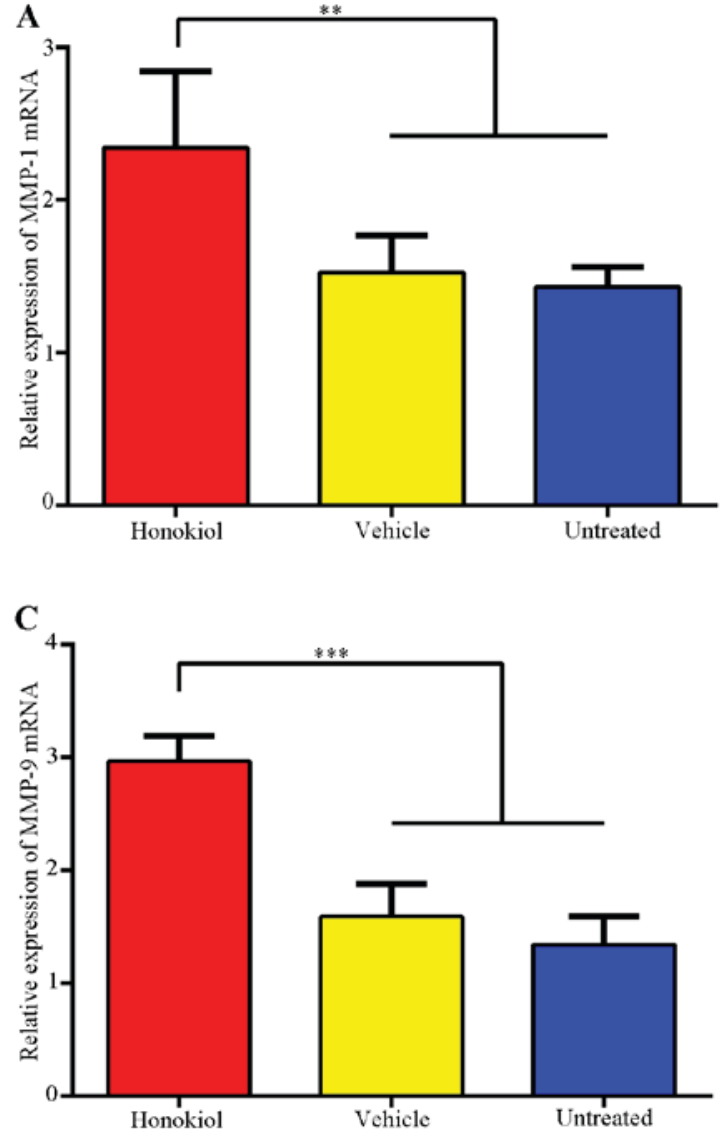

B

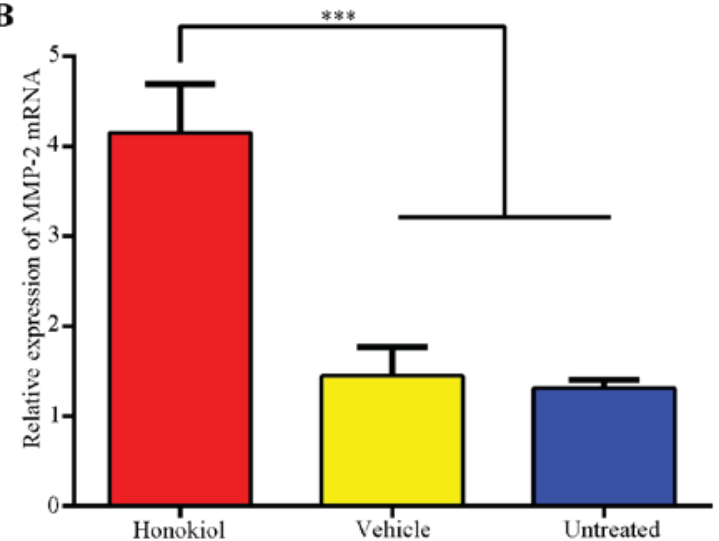

D

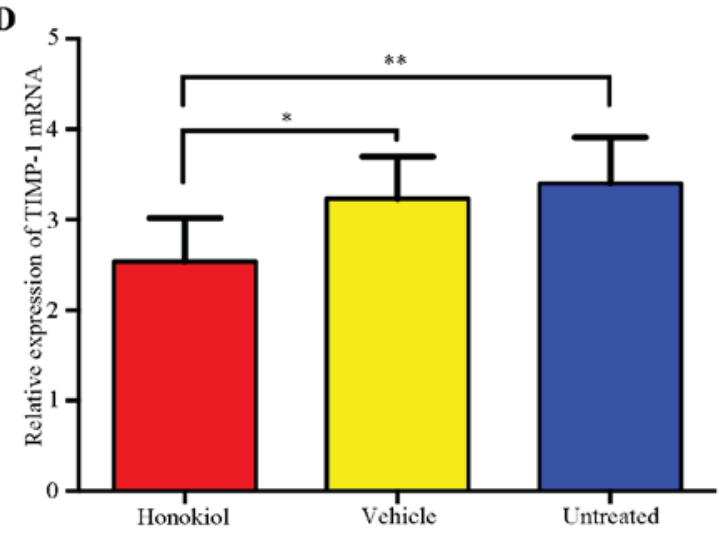

Figure 4. Effects of perivascular honokiol application on the expression of MMPs and TIMP-1. (A) Reverse transcription-quantitative polymerase chain reaction analysis of (A) MMP-1, (B) MMP-2, (C) MMP-9 and (D) TIMP-1 mRNA expression, GAPDH was used as an internal standard (n=8). $\mathrm{P}<0.05$, ${ }^{* *} \mathrm{P}<0.01$, ${ }^{* * * *} \mathrm{P}<0.001$. MMP-1,-2,-9, matrix metalloproteinase-1,-2,-9; TIMP-1, tissue inhibitor of metalloproteinase-1.

A

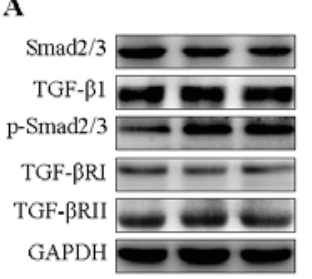

B

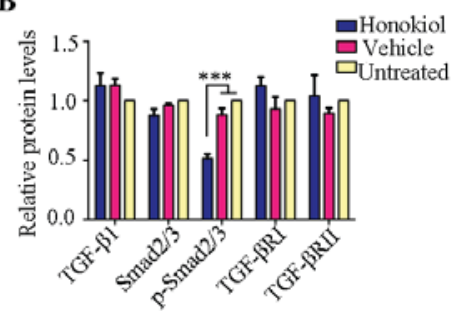

Figure 5. Effect of perivascular honokiol application on the expression of the TGF- $\beta 1 / \mathrm{Smad} 2 / 3$ signaling pathway. (A) Representative western blotting indicating the levels of Smad2/3, TGF- $\beta 1, p-S m a d 2 / 3$, TGF- $\beta$ RI and TGF- $\beta$ RII. (B) Quantitative analysis of protein expression levels $(n=8)$. ${ }^{* * * *} \mathrm{P}<0.001$. Smad $2 / 3$, SMAD family member $2 / 3 ; \mathrm{p}-\mathrm{Smad} 2 / 3$, phosphorylated Smad2/3; TGF- $\beta 1$, transforming growth factor- $\beta 1$; TGF- $\beta$ RI, TGF- $\beta$ receptor type I; TGF- $\beta$ II, TGF- $\beta$ receptor type II.

the arteries 14 days after vascular injury were assessed with Masson staining (Fig. 3A), as presented in Fig. 3B, honokiol treatment group had a significantly reduced the collagen content compared with the vehicle and untreated groups, the vehicle treatment group exhibited similar collagen deposition compared with the untreated group.

Effects of perivascular honokiol application on the expression of MMPs and TIMP-1. MMPs and TIMP-1 have essential roles in intimal thickening following vascular injury; therefore, the expression of MMPs and TIMP-1 was investigated using RT-qPCR analysis. As presented in Fig. 4, there was a significant increase in the MMP-1, MMP-2 and MMP-9 mRNA expression levels when the honokiol-treated group was compared with the vehicle and untreated groups. TIMP-1 mRNA expression was significantly reduced in the honokiol treated group compared with the vehicle and untreated groups. No significant difference was identified between the vehicle-treated group and untreated group in terms of the expression of MMPs and TIMP-1.

Perivascular honokiol application downregulated phosphorylation of Smad2/3 expression. TGF- $\beta 1 / \mathrm{Smad} 2 / 3$ signaling is a critical regulator in the pathological process of intimal hyperplasia and inhibition of the TGF- $\beta 1 / \mathrm{Smad} 2 / 3$ signaling pathway may reduce VSMC proliferation and collagen deposition, thus attenuating intimal hyperplasia (15-17). Therefore, the present study investigated whether the reduced VSMC proliferation and collagen deposition were mediated via TGF- $\beta 1 / \mathrm{Smad} 2 / 3$ pathway. It is of note that western blot analysis revealed that honokiol treatment significantly reduced the expression of $\mathrm{p}-\mathrm{Smad} 2 / 3$, whereas TGF- $\beta 1$ and Smad2/3 expression showed no obvious change in the honokiol treatment group compared with vehicle or untreated groups (Fig. 5). TGF- $\beta$ RII phosphorylates TGF- $\beta$ RI and then TGF- $\beta$ RI in turn phosphorylates Smad2/3 (18); therefore, the expression of TGF- $\beta$ RI and TGF- $\beta$ RII was subsequently analyzed. However, 
no significant difference was identified between the honokiol, vehicle or untreated groups (Fig. 5).

\section{Discussion}

The present study demonstrated that perivascular honokiol treatment inhibited intimal hyperplasia in rabbits 14 days after carotid artery balloon injury. The findings also revealed that honokiol treatment reduced VSMC proliferation and collagen deposition in the arteries following vascular injury. Additionally, the mRNA expression levels of MMP-1, MMP-2 and MMP-9 were upregulated, whereas TIMP-1 was downregulated in the honokiol treatment group. Subsequently the underlying mechanism was investigated and it was revealed that honokiol treatment reduced the phosphorylation of $\operatorname{Smad} 2 / 3$.

The adventitia has been considered to be an important regulator in vascular diseases, including hypertension, atherosclerosis and restenosis (19). Perivascular drug delivery is an effective method to prevent intimal hyperplasia as it may obtain controlled release and reduce the risk of thrombosis (drug released into the inner wall of vessels can impair reendothelialization, which would increase the risk of thrombosis) $(13,20)$. The present study has demonstrated that local application of honokiol around the injured carotid artery may inhibit intimal thickening.

VSMCs have an important function in intimal thickening, and inhibition of VSMC proliferation is a feasible method to prevent intimal hyperplasia (2). Collagen is the most abundant matrix protein in ECM, treatments reducing collagen deposition are also feasible options to inhibit intimal thickening (3). The present study identified that perivascular honokiol application may reduce VSMCs proliferation 14 days after vascular injury, which was consistent with previous studies in vitro $(10,11)$. Collagen content was also reduced in the honokiol-treated group compared with vehicle and untreated groups (Fig. 3). Additionally, a previous study determined that honokiol was able to reduce collagen deposition in a renal fibrosis model (21), demonstrating a potential antifibrotic effect of honokiol on other diseases.

MMPs and TIMPs have important functions in intimal thickening, MMPs may reduce ECM components and promote VSMC migration/proliferation, whereas TIMP-1 inhibits MMPs activity (22-24). It has been previously reported that honokiol may suppress the expression of MMPs in other diseases, such as cancer (melanoma, non-melanoma, urinary bladder cancer and gastric cancer) $(25,26)$ and arthritis $(27,28)$. However, the current study identified increased MMP-1, MMP-2 and MMP-9 mRNA levels in the honokiol-treated group, whereas TIMP-1 mRNA expression was significantly lower compared with vehicle or untreated groups (Fig. 4), this suggested that honokiol may increase the expression of MMPs and reduce the expression of TIMP-1 in local arteries, one of the possible reasons for this phenomenon is the difference between the cell lines and animal models; however, the underlying mechanism remains to be elucidated.

TGF- $\beta 1 / \mathrm{Smad} 2 / 3$ signaling has a key role in intimal hyperplasia; therefore, inhibition of the TGF- $\beta / \mathrm{Smad} 2 / 3$ signaling pathway may reduce VSMCs proliferation and collagen deposition, thus attenuating intimal hyperplasia (15-17). It is of note that the present study determined that honokiol treatment downregulated the protein expression level of p-Smad2/3, whereas the level of TGF- $\beta 1$ and total Smad2/3 remained constant. TGF- $\beta$ receptors may phosphorylate Smad2/3 (17); therefore, the present study investigated the expression of TGF- $\beta$ RI and TGF- $\beta$ RII. The results revealed that TGF- $\beta$ RI and TGF- $\beta$ RII were not significantly altered in the honokiol-treatment group. The findings of the present study revealed that honokiol may attenuate intimal hyperplasia by suppressing the phosphorylation of Smad2/3. A previous study determined that a natural compound baicalein may block the phosphorylation of Smad2 and Smad3 in hypertrophic scar-derived fibroblasts; however, baicalein did not affect the expression of TGF- $\beta 1$, Smad2/3, TGF- $\beta$ RI and TGF- $\beta$ RII, the previous study determined that underlying mechanism involved baicalein binding directly to the catalytic region of activin receptor-like kinase 5 (ALK5) to inhibit phosphorylation of Smad2 and Smad3 (29). It is possible that in the present study honokiol blocked the phosphorylation of $\mathrm{Smad} 2 / 3$ via a pathway that did not involve any change in TGF- $\beta 1 / \mathrm{Smad} 2 / 3$ signaling, similar to ALK5.

In summary, the present study revealed that perivascular honokiol application reduced intimal thickening through inhibiting VSMCs proliferation and reducing collagen deposition in rabbits 14 days after carotid artery injury. Therefore, honokiol may potentially in reduce intimal hyperplasia in the pathological process of vascular disorders. To the best of our knowledge, the current study is the first to determine that honikiol may increase the expression of MMP-1, MMP-2 and MMP-9 and reduce TIMP-1 expression in rabbit arteries. Additionally, perivascular honokiol application inhibited intimal thickening, most likely through inhibiting the phosphorylation of Smad2/3.

\section{Acknowledgements}

The present study was supported by the National Natural Science Foundation of China for extending financial support (project no. 81300092).

\section{References}

1. Forte A, Rinaldi B, Berrino L, Rossi F, Galderisi U and Cipollaro M: Novel potential targets for prevention of arterial restenosis: Insights from the pre-clinical research. Clin Sci (Lond) 127: 615-634, 2014.

2. Dzau VJ, Braun-Dullaeus RC and Sedding DG: Vascular proliferation and atherosclerosis: New perspectives and therapeutic strategies. Nat Med 8: 1249-1256, 2002.

3. Osherov AB, Gotha L, Cheema AN, Qiang B and Strauss BH: Proteins mediating collagen biosynthesis and accumulation in arterial repair: Novel targets for anti-restenosis therapy. Cardiovasc Res 91: 16-26, 2011.

4. Kumar A, Kumar Singh U and Chaudhary A: Honokiol analogs: A novel class of anticancer agents targeting cell signaling pathways and other bioactivities. Future Med Chem 5: 809-829, 2013.

5. Pan J, Lee Y, Wang Y and You M: Honokiol targets mitochondria to halt cancer progression and metastasis. Mol Nutr Food Res 60: 1383-1395, 2016

6. Hu H, Zhang XX, Wang YY and Chen SZ: Honokiol inhibits arterial thrombosis through endothelial cell protection and stimulation of prostacyclin. Acta Pharmacol Sin 26: 1063-1068, 2005.

7. Zhang GS, Wang RJ, Zhang HN, Zhang GP, Luo MS and Luo JD: Effects of chronic treatment with honokiol in spontaneously hypertensive rats. Biol Pharm Bull 33: 427-431, 2010. 
8. Fetalvero KM, Martin KA and Hwa J: Cardioprotective prostacyclin signaling in vascular smooth muscle. Prostaglandins Other Lipid Mediat 82: 109-118, 2007.

9. Patel SD, Waltham M, Wadoodi A, Burnand KG and Smith A The role of endothelial cells and their progenitors in intima hyperplasia. Ther Adv Cardiovasc Dis 4: 129-141, 2010.

10. Lee B, Kim CH and Moon SK: Honokiol causes the p21WAF1-mediated G(1)-phase arrest of the cell cycle through inducing p38 mitogen activated protein kinase in vascular smooth muscle cells. FEBS Lett 580: 5177-5184, 2006.

11. Fan S, Li X, Lin J, Chen S, Shan J and Qi G: Honokiol inhibits tumor necrosis factor- $\alpha$-stimulated rat aortic smooth muscle cel proliferation via caspase- and mitochondrial-dependent apoptosis. Inflammation 37: 17-26, 2014

12. Seedial SM, Ghosh S, Saunders RS, Suwanabol PA, Shi X, Liu B and Kent KC: Local drug delivery to prevent restenosis. J Vasc Surg 57: 1403-1414, 2013.

13. Chaudhary MA, Guo LW, Shi X, Chen G, Gong S, Liu B and Kent KC: Periadventitial drug delivery for the prevention of intimal hyperplasia following open surgery. J Control Release 233: 174-180, 2016.

14. Livak KJ and Schmittgen TD: Analysis of relative gene expression data using real-time quantitative PCR and the 2(-Delta Delta C(T)) method. Methods 25: 402-408, 2001

15. Chen W, Chu Y, Zhu D, Yan C, Liu J, Ji K and Gao P. Perivascular gene transfer of dominant-negative N19RhoA attenuates neointimal formation via inhibition of TGF-beta1-Smad2 signaling in rats after carotid artery balloon injury. Biochem Biophys Res Commun 389: 217-223, 2009.

16. Suwanabol PA, Kent KC and Liu B: TGF- $\beta$ and restenosis revisited: A Smad link. J Surg Res 167: 287-297, 2011.

17. Lu P, Wang S, Cai W and Sheng J: TGF-beta $1 / \mathrm{Smad} 3$ expression and its effects on carotid intimal hyperplasia. Front Biosci (Elite Ed) 4: 2022-2028, 2012

18. Kamato D, Burch ML, Piva TJ, Rezaei HB, Rostam MA, Xu S Zheng W, Little PJ and Osman N: Transforming growth factor- $\beta$ signalling: Role and consequences of Smad linker region phosphorylation. Cell Signal 25: 2017-2024, 2013.

19. Sartore S, Chiavegato A, Faggin E, Franch R, Puato M, Ausoni S and Pauletto P: Contribution of adventitial fibroblasts to neointima formation and vascular remodeling: From innocent bystander to active participant. Circ Res 89: 1111-1121, 2001.

20. Luscher TF, Steffel J, Eberli FR, Joner M, Nakazawa G, Tanner FC and Virmani R: Drug-eluting stent and coronary thrombosis: Biological mechanisms and clinical implications. Circulation 115: 1051-1058, 2007.
21. Chiang CK, Sheu ML, Lin YW, Wu CT, Yang CC, Chen MW, Hung KY, Wu KD and Liu SH: Honokiol ameliorates renal fibrosis by inhibiting extracellular matrix and pro-inflammatory factors in vivo and in vitro. Br J Pharmacol 163: 586-597, 2011.

22. Newby AC: Dual role of matrix metalloproteinases (matrixins) in intimal thickening and atherosclerotic plaque rupture. Physiol Rev 85: 1-31, 2005

23. Azevedo A, Prado AF, Antonio RC, Issa JP and Gerlach RF Matrix metalloproteinases are involved in cardiovascular diseases. Basic Clin Pharmacol Toxicol 115: 301-314, 2014.

24. Jacob MP: Extracellular matrix remodeling and matrix metalloproteinases in the vascular wall during aging and in pathological conditions. Biomed Pharmacother 57: 195-202, 2003.

25. Ahn KS, Sethi G, Shishodia S, Sung B, Arbiser JL and Aggarwal BB: Honokiol potentiates apoptosis, suppresses osteoclastogenesis, and inhibits invasion through modulation of nuclear factor-kappaB activation pathway. Mol Cancer Res 4: 621-633, 2006

26. Zhang Q, Zhao W, Ye C, Zhuang J, Chang C, Li Y, Huang X, Shen L, Li Y, Cui Y, et al: Honokiol inhibits bladder tumor growth by suppressing EZH2/miR-143 axis. Oncotarget 6: 37335-37348, 2015.

27. Kim KR, Park KK, Chun KS and Chung WY: Honokiol inhibits the progression of collagen-induced arthritis by reducing levels of pro-inflammatory cytokines and matrix metalloproteinases and blocking oxidative tissue damage. J Pharmacol Sci 114: 69-78, 2010

28. Chen YJ, Tsai KS, Chan DC, Lan KC, Chen CF, Yang RS and Liu SH: Honokiol, a low molecular weight natural product, prevents inflammatory response and cartilage matrix degradation in human osteoarthritis chondrocytes. J Orthop Res 32: 573-580, 2014.

29. Zhang YF, Zhou SZ, Cheng XY, Yi B, Shan SZ, Wang J and Li QF: Baicalein attenuates hypertrophic scar formation via inhibition of the transforming growth factor- $\beta / \mathrm{Smad} 2 / 3$ signalling pathway. Br J Dermatol 174: 120-130, 2016.

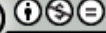

This work is licensed under a Creative Commons Attribution-NonCommercial-NoDerivatives 4.0 International (CC BY-NC-ND 4.0) License. 